\title{
Características Atentivas Reveladas por Dicas Múltiplas Locais e Globais ${ }^{1}$
}

\author{
Cesar Galera² \\ Mikael Cavallet \\ Universidade de São Paulo - Ribeirão Preto \\ Michael von Grünau \\ Afroditi Panagopoulos \\ Concordia University
}

\begin{abstract}
RESUMO - As características do foco atentivo foram investigadas por meio do efeito de dicas locais múltiplas e globais, múltipla e unitária, sobre o desempenho em uma tarefa de busca visual. Os resultados mostram um ganho no desempenho de provas com dicas locais múltiplas válidas; o ganho é proporcional ao tamanho da área na qual os estímulos são apresentados. Nas provas com dica local válida para área existe ganho quando os estímulos são apresentados na área maior e custo na área menor. Dicas globais não proporcionam ganho em provas com dicas válidas, mas são acompanhadas de custo nas provas com dicas inválidas. Também existe custo nas provas em que o foco atentivo deve ser expandido ou contraído. Os resultados sugerem que o modelo de focos atentivos múltiplos é mais adequado para explicar o desempenho em tarefas com dicas locais múltiplas.
\end{abstract}

Palavras-chave: percepção visual; atenção visual; dicas locais múltiplas; dicas globais.

\section{Attentive Characteristics Revealed by Local and Global Multiple Cues}

\begin{abstract}
The characteristics of the attentive focus were investigated through the effect of local and global multiple cues, multiple and unitary, on the performance of a visual search task. The results show a gain in the performance of tests with valid multiple local cues; the gain is proportional to the size of the area in which the stimuli are presented. In the tests with valid local tips for the area there is a gain when the stimuli are presented in the larger area and a cost in the smaller area. Global cues do not generate a gain in tests with valid cues but are followed by a cost in tests with invalid cues. There is also a cost in tests where the attentive focus must be expanded or contracted. The results suggest that the model of multiple attentive focuses is more adequate to explain performance in tests with multiple local cues.
\end{abstract}

Key words: visual perception; visual attention; multiple local cues; global cues..

A seleção da informação visual tem sido descrita como análoga ao foco de uma lanterna que se desloca por um quarto escuro (Posner, Snyder \& Davidson, 1980). Os objetos iluminados são processados de forma privilegiada, enquanto que objetos localizados na penumbra são ignorados. Em outra analogia a atenção visual é equiparada ao zum de uma lente que pode ser ajustado ou ampliado em função das necessidades impostas por tarefas específicas (Eriksen \& St. James, 1986; Eriksen \& Yeh, 1985). Nesta concepção, a concentração de recursos atentivos no interior do foco da atenção é inversamente proporcional ao seu tamanho, de forma que o desempenho será melhor quando o foco for pequeno, e tenderá a degradar na medida em que os recursos atentivos se distribuem por uma área maior.

No paradigma clássico utilizado por Posner (1980) a posição na qual um estímulo pode ser apresentado é informada ao sujeito através de uma dica (cue). O estímulo

Financiamento: CNPq, CAPES (CG), NSERC, FCAR (MvG).

2 Endereço: FFCLRP- USP, Av. Bandeirantes 3900, Ribeirão Preto, São Paulo, SP, Brasil, 14040-901. Email: cesar_galera@ffclrp.usp.br apresentado na posição indicada é processado com maior eficiência do que aquele apresentado em local não indicado. Este tipo de resultado sugere que estímulos apresentados nas posições indicadas são submetidos a um processamento privilegiado, enquanto que estímulos apresentados fora do local indicado têm seu processamento atrasado pela necessidade de se deslocar o foco atentivo para posição do estímulo. Embora diferentes nos detalhes, ambos os modelos de foco único concordam em dois pontos básicos. Primeiro, consideram que a atenção visual opera com base na representação espacial do ambiente, e segundo, consideram que o foco atentivo é unitário e indivisível, não sendo possível prestar atenção a posições espaciais distintas simultaneamente (Cave \& Bichot, 1999; LaBerge \& Brown, 1989).

Quando duas ou mais dicas espaciais são apresentadas simultaneamente, existem sugestões de que o foco atentivo se expande, abarcando não só os vários locais indicados, mas também as posições intermediárias entre eles (Eriksen \& St. James, 1986; Eriksen \& Yeh, 1985). Vários estudos têm corroborado essa suposição. Por exemplo, Heinze e colaboradores (1994) compararam potenciais evocados de curta latência (p1) quando seus sujeitos atendiam a duas formas geométricas apresentadas em locais contíguos ou separados por outra 
forma geométrica. Seus resultados mostram uma diminuição na latência dos potenciais evocados por estímulos apresentados nos locais indicados e também para estímulos apresentados entre os locais indicados, sugerindo que a região entre os alvos também havia sido selecionada.

Por outro lado, vários estudos têm sugerido que o foco atentivo pode ser dirigido a regiões não contíguas. Por exemplo, Kramer e Hahn (1995) investigaram se duas posições espaciais não-contíguas poderiam ser seletivamente atendidas num experimento no qual duas áreas separadas do campo visual foram indicadas. A tarefa dos sujeitos era identificar letras-alvo apresentadas nessas posições, enquanto distratores eram apresentados entre as regiões indicadas. Os autores supunham que os distratores não teriam nenhum efeito no desempenho, caso o foco atentivo pudesse ser dividido entre as duas posições indicadas, sem levar em conta a região entre elas. De fato, os sujeitos podiam ignorar o distrator quando este e as letras-alvo eram revelados de maneira gradual, mas não quando eram apresentados subitamente, sugerindo que a atenção pode selecionar áreas não-contíguas no campo visual. Bahcall e Kowler (1999) mostram que estímulos apresentados em locais previamente indicados recebem processamento privilegiado sugerindo que os recursos atencionais podem ser divididos em diferentes pontos no espaço. Os estudos de Scharlau (2004) também mostram que o julgamento da ordem temporal de dois estímulos, apresentados em posições separadas no espaço, não é afetado pela presença de um distrator apresentado numa posição intermediária, sugerindo que as posições indicadas podem ser simultaneamente atendidas, enquanto se ignora uma posição intermediária.

Também na tarefa de busca visual existem evidências de que é possível atender simultaneamente a vários locais nãocontíguos. No estudo de Bichot, Cave e Pashler (1999), os sujeitos foram instruídos a prestar atenção a dígitos vermelhos apresentados entre dígitos verdes. Independente de os estímulos serem apresentados isoladamente, ou em pares, o desempenho foi o mesmo, sugerindo que a atenção pôde ser dividida simultaneamente entre duas posições, sem levar em conta os estímulos localizados entre as regiões atendidas. O estudo de Galera, Lopes e von Grünau (2001) também mostra que os estímulos relevantes para a tarefa de busca visual podem ser selecionados e analisados independente da presença de elementos de fundo, irrelevantes para a tarefa.

De acordo com Wright e colaboradores (Richard, Wright \& Ward, 2003; Wright, 1994; Wright \& Richard, 2003) é possível conciliar a existência de um foco atentivo único com os ganhos obtidos em situações nas quais um alvo pode ser apresentado em várias posições indicadas por dicas múltiplas. De acordo com esta concepção, o aparecimento abrupto de estímulos no campo visual desencadeia, simultaneamente nos locais em que os estímulos são apresentados, uma forma de processamento sensorial que pode influenciar a resposta a alvos apresentados nesses mesmos locais. Este processo de ativação sensorial é pré-atentivo, no sentido em que fornece aos processos atentivos a informação sobre a localização de eventos recentes que podem ou devem ser examinados. $\mathrm{O}$ foco atencional pode se abrir em diferentes posições do campo, de maneira serial, em função das diferenças na magnitude da ativação sensorial. No caso em que várias posições são indicadas simultaneamente pelo aparecimento abrupto de dicas múltiplas, o processamento dos estímulos apresentados subseqüentemente nas mesmas posições será beneficiado pela ativação sensorial produzida pelas dicas, assim como pela ativação top-down, determinada pelas expectativas e pelos objetivos mais imediatos do sujeito.

Neste estudo investigamos a focalização da atenção visual utilizando o paradigma de dicas múltiplas para estímulos de uma tarefa de busca visual. Neste paradigma as dicas espaciais informam as posições nas quais os estímulos relevantes para uma tarefa de busca visual, alvo e distratores, poderão ser apresentados. De acordo com o modelo de foco atentivo único de tamanho flexível, a apresentação de dicas múltiplas dá início a um processo de expansão do foco atencional de forma a cobrir todas as dicas. Estímulos apresentados entre as posições indicadas serão processados tão rapidamente quanto os estímulos apresentados exatamente nessas posições, pois também estarão localizados sob foco atentivo. De acordo com esse modelo devemos obter um ganho no desempenho nas provas em que as dicas informam corretamente as posições nas quais serão apresentados os estímulos da tarefa de busca (dicas válidas) e nas provas em que os estímulos da tarefa de busca visual sejam apresentados entre as posições indicadas (dica válida para área). De acordo com o modelo de ativação sensorial e com o modelo de dicas múltiplas deveremos obter ganho nas provas com dicas válidas, e custo nas provas com dicas válidas para área. De acordo com ambos os modelos, os sujeitos deverão ser capazes de selecionar de forma mais eficiente os estímulos apresentados nas posições indicadas, ignorando os estímulos apresentados nas posições intermediárias. Além disso, o custo da seleção de estímulos apresentados nas posições intermediárias àquelas indicadas deve ser maior quando os estímulos forem apresentados de maneira gradual (Experimento 2) do que quando apresentados de maneira abrupta (Experimento 1).

\section{EXPERIMENTO 1}

\section{Método}

\section{Participantes}

Oito participantes e um autor (CG) fizeram parte deste experimento, todos com visão normal (20/20) ou corrigida. Os participantes receberam uma pequena recompensa $(\mathrm{R} \$ 5,00)$ para participar de uma sessão experimental com duração de 50 minutos.

\section{Materiais}

$\mathrm{O}$ experimento foi conduzido com o programa E-Prime versão 1.0 (Schneider, Eschman \& Zuccoloto, 2002). Os estímulos relevantes para a tarefa de busca visual, um alvo e três distratores, eram letras "T" apresentadas em diferentes orientações. $\mathrm{O}$ alvo era um $\mathrm{T}$ orientado $90^{\circ}$ para a direita $(\dashv)$ ou para a esquerda $(\vdash)$. Os distratores eram 
Ts apresentados na posição normal ( $\mathbf{T}$ ) ou invertida ( $\perp$ ). Os elementos de fundo eram letras T, orientadas em $45^{\circ}$ e $135^{\circ}$ para a esquerda ou para a direita (respectivamente $\boldsymbol{K}, \boldsymbol{\lambda} \mathrm{e} \boldsymbol{\nearrow}, \boldsymbol{Y}$ ). Os segmentos verticais e horizontais dos Ts subtendiam $0,8^{\circ}$ de ângulo visual de comprimento por $0,1^{\circ}$ de largura, apresentados em preto $\left(0.9 \mathrm{~cd} / \mathrm{m}^{2}\right)$ sobre o fundo branco $\left(70 \mathrm{~cd} / \mathrm{m}^{2}\right)$ da tela de um monitor Flatron LG com resolução de 800 × 600 pixels. A dica era formada por quatro pontos pretos com $0,8^{\circ}$ de diâmetro. $O$ ponto de fixação era uma cruz com braços de $0,6^{\circ}$ apresentada no centro da tela. Dica e estímulos relevantes para a tarefa de busca foram apresentados em quatro configurações espaciais grandes e quatro pequenas (Figura 1). Nas configurações grandes, esses estímulos foram apresentados, em média, a $3,9^{\circ}$ do ponto central de fixação. Na configuração pequena, os estímulos foram apresentados, em média, a $2,3^{\circ}$ do ponto de fixação. Os participantes permaneceram sentados em frente à tela a uma distância de aproximadamente $58 \mathrm{~cm}$.

\section{Procedimento}

Os participantes eram instruídos a fixar o olhar sobre o ponto de fixação e realizar a tarefa de busca visual respondendo o mais rápido possível no teclado numérico do computador. A tecla 1 do teclado numérico deveria ser pressionada caso o alvo estivesse orientado à esquerda, e a tecla

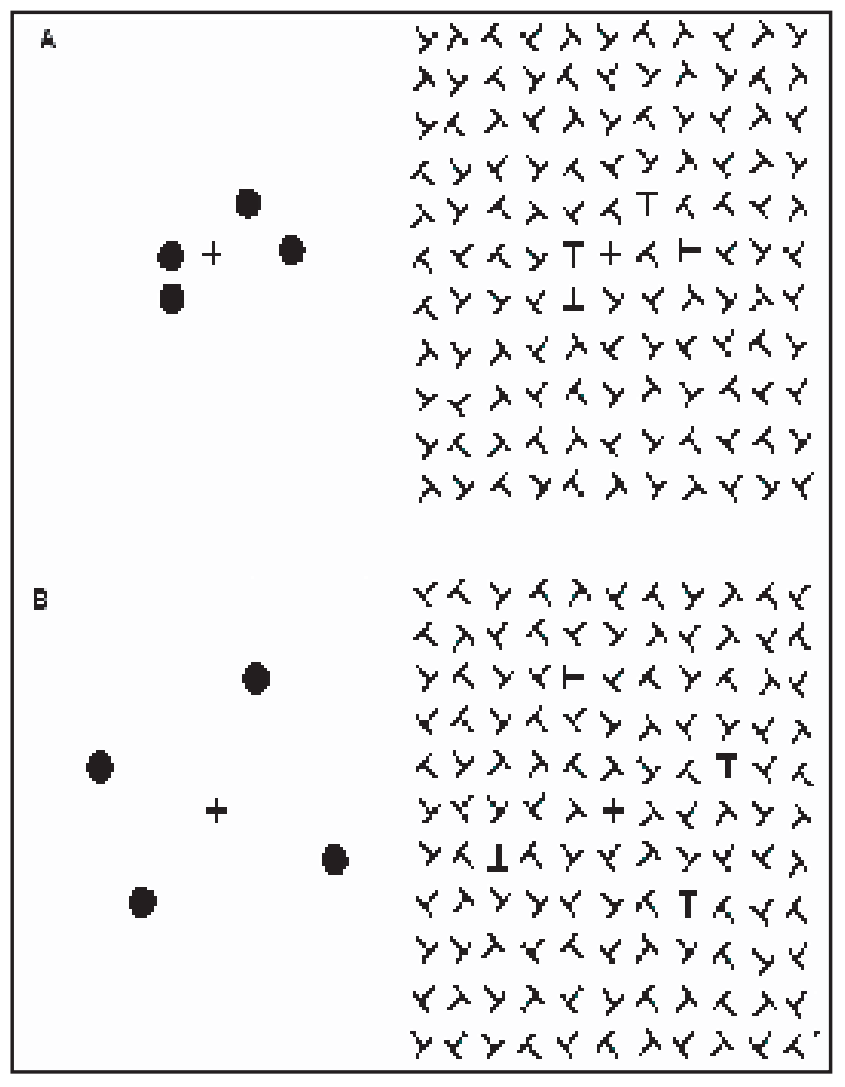

Figura 1. Exemplo de dicas (esquerda) e estímulos (direita) utilizados no Experimento 1. a) Dica válida para local, apresentada na área menor (distância média de $2,3^{\circ}$ do ponto de fixação) e. b) Dica válida para área, apresentada na área maior (a distância média de $3,9^{\circ}$ do ponto de fixação).
2, caso o alvo estivesse orientado à direita. Essas respostas eram dadas com os dedos indicador e médio da mão direita, respectivamente. Os participantes também foram instruídos sobre a natureza da informação proporcionada pelas dicas (validade). A dica local válida informava exatamente a posição na qual os estímulos relevantes seriam apresentados (73\% das provas). A dica poderia ser válida somente para a área na qual seriam apresentados os estímulos $(9,1 \%$ das provas), caso em que dica e estímulos eram apresentados entre as posições indicadas, com nenhuma posição em comum, mas à mesma distância do ponto de fixação. A dica poderia ser inválida $(9,1 \%$ das provas), caso em que poderia ser apresentada numa configuração pequena e os estímulos numa configuração grande, ou vice-versa. Finalmente, em $9,1 \%$ das provas, na situação sem dica, um único ponto foi apresentado sobre o ponto de fixação no momento em que a dica deveria ser apresentada.

Cada prova começou com a apresentação do ponto de fixação no centro da tela por um segundo, seguido pela apresentação da dica por $96 \mathrm{~ms}$. Os estímulos relevantes para a tarefa de busca eram apresentados depois de um intervalo dica-estímulo (IDE) de 163 ou $277 \mathrm{~ms}$ e permaneciam na tela até que o participante respondesse, por um tempo máximo de 2 segundos.

Em cada sessão, as oito configurações (quatro pequenas e quatro grandes), a posição do alvo em cada configuração, a orientação do alvo (esquerda, direita), e a validade da dica (válida, válida para área, inválida e sem dica) variaram aleatoriamente de prova para prova. Em cada sessão foram realizadas 25 provas-treino, que não foram analisadas, e 704 provas-teste, $512 \mathrm{com}$ dica válida, 64 com dica válida para a área, 64 com dica inválida e 64 sem dica. As provas-teste foram divididas em dois blocos, cada um dedicado a um IDE. Dessa forma, o delineamento experimental envolveu medidas repetidas em três fatores experimentais: a validade da dica, o tamanho da área da configuração e o IDE.

\section{Resultados e Discussão}

O tempo de reação médio (TR) foi analisado por meio de uma análise de variância para medidas repetidas em três fatores: validade da dica (local válida, válida para área, inválida, sem dica), IDE (intervalo dica-estímulo de $163 \mathrm{~ms}$, $277 \mathrm{~ms}$ ) e tamanho da área da configuração (grande, pequena). Um resumo dos TRs é apresentado na Figura 2. A validade da dica afetou o TR de forma significativa $(F(3$, $24)=8,31 ; p=0,001)$. O teste de Newman-Keuls $(p<0,05)$ mostrou que a dica válida para local e a dica válida para área tiveram um efeito benéfico no desempenho (619 e 636ms, respectivamente) quando comparadas com a situação sem dica (654ms), enquanto que o custo associado à dica inválida para área $(659 \mathrm{~ms})$ não foi significativo. $\mathrm{O}$ efeito principal do tamanho da área da configuração não foi significativo $(p=0,09)$, no entanto este fator interage com a validade da dica $(F(3,24)=4,00 ; p=0,019)$. A informação válida para área beneficiou os estímulos apresenta- 
dos na configuração grande $(642 \mathrm{~ms})$ em relação àqueles apresentados nas provas sem dica $(666 \mathrm{~ms})$ ou em relação àqueles apresentados em provas com dicas inválidas (686ms), ou seja, neste caso houve um custo significativo quando os estímulos foram apresentados numa configuração maior do que a configuração da dica. Nas provas realizadas na área menor, apenas os estímulos precedidos de dicas válidas $(603 \mathrm{~ms})$ foram beneficiados em relação aos estímulos que se seguiram às dicas válidas para área (629ms), dicas inválidas $(631 \mathrm{~ms})$ e em relação a aqueles que foram apresentados sem dicas $(642 \mathrm{~ms})$, e neste caso não houve nenhum custo significativo quando os estímulos foram apresentados numa configuração menor do que aquela indicada.

De maneira geral o desempenho é melhor $(627 \mathrm{~ms})$ quando o IDE é longo do que quando é curto (663ms) $(F(1,8)=13,01, p=0,007)$. Além disso o IDE afeta a interação entre a validade da dica e o tamanho da configuração $(F(3,24)=5,58, p=0,005)$. A interação entre estes fatores mostra que o efeito da validade da dica foi maior para o intervalo mais curto. No intervalo de $163 \mathrm{~ms}$, a dica inválida para área causou um custo significativo quando a configuração dos estímulos era maior do que a configuração da dica, e teve um efeito benéfico pequeno, marginal, quando a configuração dos estímulos-teste era menor do que a configuração da dica. No intervalo mais longo, estes efeitos foram similares, mas não foram estatisticamente significativos.

A porcentagem de erros cometidos em cada tratamento foi submetida à mesma análise que o TR. Existe apenas uma tendência a serem cometidos mais erros nas provas com intervalo dica-estímulo curto $(6,2 \%)$ do que nas provas com intervalos longos $(3,4 \%)(p=0,06)$. Nenhum outro fator ou interação teve efeitos significativos sobre a taxa de erros.

A dica local válida e a dica válida para área tiveram efeitos benéficos sobre o desempenho. Uma tendência de ganho mais nítida ocorreu para a dica local válida comparado àquele obtido com a dica válida para área. A informa-

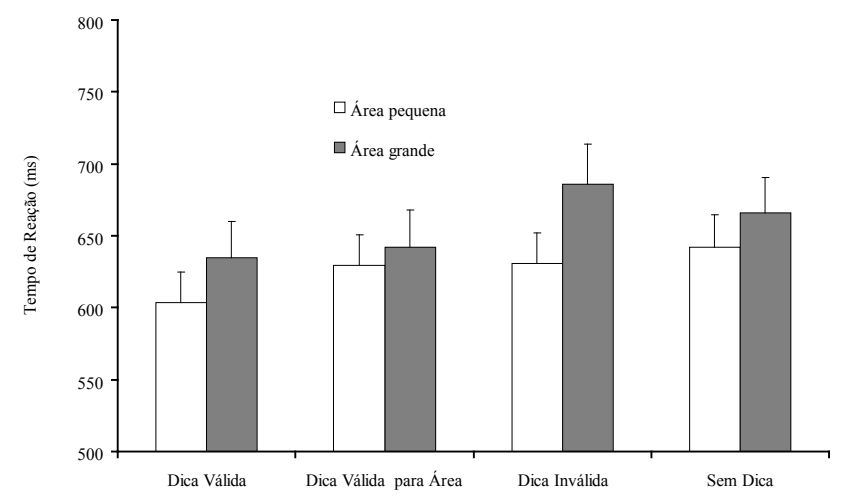

Figura 2. Experimento 1:. TRs médios (e erro padrão da média) em função do tamanho da área na qual foram apresentados os estímulos da tarefa de busca nas condições com Dica Válida, Dica Válida para Área, Dica Inválida e Sem Dica. ção sobre a área onde os estímulos-teste seriam apresentados também foi importante para o desempenho. Os estímulos apresentados nas posições intermediárias àquelas indicadas também são beneficiados, sugerindo que a dica, além da informação sobre a localização específica, tem um efeito global que poderia ser mais facilmente explicado por um modelo de foco atentivo único de tamanho flexível do que pela existência de focos atencionais múltiplos, ou do que pela proposta de um foco único que se desloca com base na informação proporcionada pela indexação das posições indicadas.

Este efeito global também é sugerido nas provas em que os estímulos são apresentados numa área diferente do que a indicada. Neste caso, dependendo do IDE, a dica inválida para área causa tanto um custo como um benefício, dependendo da diferença entre o tamanho da área indicada e da área coberta pela configuração de estímulos teste. Há um custo se os estímulos teste forem apresentados em uma configuração maior do que aquela indicada, e há um benefício se os estímulos teste forem apresentados em uma configuração que seja menor do que aquela indicada. Estes resultados sugerem que o tamanho do foco atentivo deve ser escalonado até a área a ser analisada apenas se o foco for menor do que a área onde os estímulos da tarefa de busca aparecem. Se o foco atentivo já for maior do que a área relevante, não haverá necessidade de focalizar a área menor.

\section{EXPERIMENTO 2}

Ao contrário do Experimento 1, no qual as dicas eram apresentadas num campo visual vazio, neste experimento as dicas foram apresentadas de maneira gradual num campo visual preenchido por marcadores de posição, pequenos quadrados com quatro caselas internas, formados pela sobreposição de quatro Ts. Através da retirada de segmentos específicos, os marcadores eram transformados em estímulos relevantes ou estímulos de fundo. Vários estudos têm mostrado que a captura da atenção é menos eficiente quando os estímulos são apresentados desta maneira (Yantis \& Jonides, 1984). A apresentação gradual dos estímulos foi utilizada neste estudo para minimizar o processo de ativação sensorial que a apresentação abrupta de distratores e estímulos apresentados em posições intermediárias àquelas indicadas poderia causar. Desta forma, espera-se um aumento da ativação nos locais indicados, acompanhado de um ganho maior para estímulos apresentados nessas posições, em comparação com estímulos apresentados em posições intermediárias.

Além dessa mudança na forma de apresentação dos estímulos, procuramos explorar as diferenças no processamento da informação local e global sobre o desempenho da tarefa de busca. Com esse objetivo, provas com dicas válidas para locais e dicas válidas para área foram apresentadas em blocos separados. Dessa forma pudemos avaliar o efeito da informação sobre os locais em que seriam apresentados os estímulos, com a informação mais global sobre a área na 
qual seriam apresentados os estímulos. O bloco de provas com dica local tem as mesmas características básicas das provas com dica válida e dica válida para área do experimento anterior. Neste bloco foram apresentadas provas em que o participante era informado sobre a localização exata na qual seriam apresentados os estímulos (dica local válida) e provas nas quais os estímulos eram apresentados entre as posições indicadas, mas à mesma distância do ponto de fixação (dicas válidas para área).

Em um segundo bloco, com dica múltipla para área, foram replicadas as provas com dica válida para área $\mathrm{e}$ provas com dicas inválidas do experimento anterior. Neste bloco de provas, a dica válida para área informava aos sujeitos apenas a área, mas não a posição exata em que os estímulos relevantes seriam apresentados. Embora proporcionada pelos mesmos estímulos, a informação neste bloco de provas foi considerada como global, uma vez que os estímulos da tarefa de busca eram apresentados entre os locais indicados (dica válida para área) ou na área diferente daquela indicada (dica inválida). Num terceiro bloco de provas, uma dica global única informava aos participantes apenas a área na qual seriam apresentados os estímulos e, como nos outros blocos, esta informação poderia ser válida ou não.

De acordo com um modelo atentivo de foco único com tamanho flexível, dicas locais, que informem com precisão as posições nas quais os estímulos relevantes serão apresentados, devem proporcionar um ganho no desempenho, tanto nas provas com dicas válidas, como nas provas com dicas válidas para área, nas quais os estímulos são apresentados em posições intermediárias àquelas indicadas. De acordo com o mesmo modelo, uma dica global (múltipla ou única), que informe apenas a região na qual serão apresentados os estímulos, também deve proporcionar um ganho equivalente no desempenho, tanto nas provas com dicas válidas como nas provas inválidas nas quais os estímulos são apresentados no interior da região indicada. Nas provas inválidas nas quais a área coberta pela dica for menor do que a área coberta pelos estímulos relevantes deveria ocorrer um custo associado à expansão do foco atentivo.

Os resultados do Experimento 1 e resultados parciais apresentados no experimento $2 \mathrm{~A}$ sugeriram que os efeitos da validade da dica poderiam ser mais acentuados com IDEs mais curtos. Dessa forma os IDEs foram reduzidos no experimento $2 \mathrm{~B}$.

\section{Método}

\section{Participantes}

Tomaram parte deste estudo 21 participantes e dois dos autores (CG e MC). Um grupo com 14 participantes, incluindo os dois autores, realizou o experimento $2 \mathrm{~A}$ e um grupo com nove participantes diferentes realizou o experimento 2B. Os participantes receberam uma pequena retribuição $(\mathrm{R} \$ 5,00)$ para participar de uma sessão experi- mental com duração de aproximadamente 40 minutos.

\section{Materiais}

O mesmo equipamento e os estímulos da tarefa de busca usados no experimento anterior foram utilizados neste experimento. Sessenta e quatro quadrados com $0,7^{\circ} \times 0,7^{\circ}$ sobrepostos a um sinal de soma foram utilizados como marcadores de posição. Estes estímulos foram organizados em quatro círculos concêntricos com raios de $2^{\circ}, 4^{\circ}, 5,5^{\circ}$ e $7,5^{\circ}$ em torno do ponto de fixação. Os estímulos relevantes para a tarefa de busca e a dica eram definidos pela remoção de segmentos ou pelo preenchimento dos espaços vazios de cada marcador. Foram utilizados três tipos de dicas: local, global única e global múltipla. As dicas local e global múltipla foram dadas através de quatro quadrados pretos, a dica global única foi definida por um círculo cinza $\left(37,30 \mathrm{~cd} / \mathrm{m}^{2}\right)$ com raio de $2,5^{\circ}$ ou $6,25^{\circ}$ (Figura 3 ).

\section{Procedimento}

Os experimentos $2 \mathrm{~A}$ e $2 \mathrm{~B}$ utilizam o mesmo procedimento, exceto pelos intervalos entre dica e estímulos. Assim como no experimento anterior, o participante realizava uma tarefa de busca visual cujos estímulos eram apresentados entre elementos de fundo. Nas provas com dica válida, $60 \%$ das provas, o sujeito era informado sobre as posições exatas (dica local), ou da área na qual seriam apresentados os estímulos relevantes para a tarefa de busca (dica global múltipla e global única). Em 20\% das provas, os estímulos eram apresentados entre as posições indicadas (dica local válida para área), ou num círculo diferente daquele indicado (global múltipla inválida e global única inválida). Nas provas sem dica, $20 \%$ das provas, um quadrado preto, com $0,7^{\circ}$ de lado, era apresentado no centro da tela no momento em que

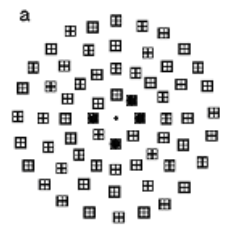

d

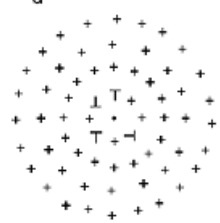

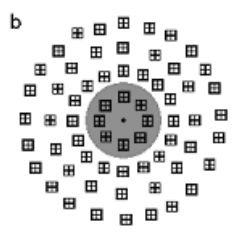

e

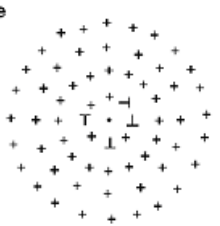

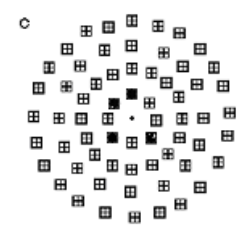

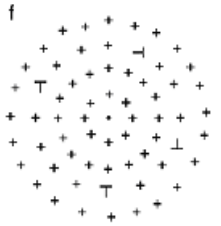

Figura 3. Exemplos de dicas e estímulos utilizados no Experimento 2. O exemplo de configuração de dica local para posição em 3a foi utilizado com dica válida para estímulos apresentados em 3e (mesmas posições), e válidas apenas para a área para os estímulos apresentados em 3d (mesma área). A dica global apresentada em 3 b é uma dica válida para estímulos como os apresentados em áreas pequenas (3d e 3e) e inválida para estímulos apresentados em áreas maiores, tal como 3f. A dica 3c, é uma dica local para área, é válida para estímulos apresentados na mesma área, como 3e, e inválida para estímulos como 3f. Esta dica aponta apenas a área na qual serão apresentados os estímulos, mas não sua posição exata. Dessa forma, a dica em $3 c$ jamais seria seguida por uma configuração de estímulos em $3 d$. 
deveriam ser apresentadas as dicas.

Cada prova tinha início com a apresentação do ponto de fixação, que permanecia no centro da tela até o final da prova. Depois de 500ms , 64 estímulos marcadores de posição eram apresentados por 500ms. A dica era então apresentada, por $120 \mathrm{~ms}$, sobreposta aos marcadores, nos locais onde poderiam ser apresentados os estímulos relevantes. Depois da apresentação da dica, os marcadores de posição permaneciam na tela por um intervalo de tempo variável (IDE de 170, 270 e 400ms no Experimento 2A, ou 50, 100, 150, no Experimento 2B), e então eram substituídos pelos estímulos relevantes e por 60 estímulos em forma de cruz. Estes estímulos permaneciam na tela por $120 \mathrm{~ms}$. Após a apresentação dos estímulos, a tela permanecia em branco por um tempo limite de $1850 \mathrm{~ms}$ para que o participante desse a resposta. As respostas corretas foram sinalizadas pelo ponto de fixação apresentado em azul. O ponto de fixação apresentado em vermelho foi utilizado para sinalizar respostas incorretas.

O mesmo delineamento experimental foi usado nos dois experimentos (2A e B) com a única exceção do IDE que foi de 170,270 e 400ms no experimento $2 \mathrm{~A}$ e 50,100 e $150 \mathrm{~ms}$ no Experimento 2B. Três outros fatores experimentais foram manipulados intra-sujeitos: tipo de dica (local, global múltipla e global única), validade da dica (válida, inválida e sem dica) e o tamanho das áreas nas quais foram apresentados os estímulos relevantes (raios de 2 e 5,5 graus de ângulo visual). Os tipos de dicas foram manipulados entre blocos de provas. Cada bloco teve início com a apresentação de 25 provas-treino, que não foram levadas em consideração na análise dos resultados. Em cada experimento os seis tratamentos resultantes das combinações entre o tamanho do círculo e a validade da dica foram apresentados 64 vezes em três blocos, cada um com 384 provas. A ordem pela qual os participantes passaram pelos diferentes tipos de dicas (blocos) foi aleatória.

\section{Resultados e Discussão}

Inicialmente os TRs das respostas corretas foram analisados considerando os dois grupos e as medidas repetidas nos fatores manipulados intra-sujeitos. Está análise mostrou que as diferenças encontradas entre os TRs dos dois grupos não são significativas $(F(1,21)=1,5 p=0,230)$, que o efeito principal do IDE é marginal $(F(2,42)=3,11 p=0,060)$, e que estes fatores também não estabelecem interação significativa entre si $(F(2,42)=1,14 p=0,330)$. Os TRs médios foram então reagrupados e submetidos a uma nova análise que levou em conta apenas o tamanho da área na qual foram apresentados os estímulos (grande, pequena), o tipo de dica (local, global múltipla e local única) e a validade da dica (válida, inválida e sem dica). Um resumo dos TRs agrupados segundo esses fatores é apresentado na Figura 4. A análise de variância mostra que o desempenho na tarefa de busca é melhor quando os estímulos são apresentados na área menor (578ms) do que quando apresentados na área maior $(651 \mathrm{~ms})(F(1,22)=131,89$, $p<0,001)$. O desempenho na tarefa de busca também é determinado pela validade da dica $(F(2,44)=66,39, p<0,001)$. $\mathrm{O}$ teste de Newman-Keuls $(p<0,05)$ permite afirmar que o desempenho é melhor nas provas com dica válida (596ms) do que nas provas sem dica $(617 \mathrm{~ms})$, e é melhor nas provas sem dicas do que nas provas com dica inválida (630ms).

Como se pode observar na Figura 4 o efeito da validade depende do tipo de dica e do tamanho da área coberta pelos estímulos $(F(4,88)=7,681, p<0,001)$. O teste de NewmanKeuls $(p<0,05)$ nos permite afirmar que nas provas com dica local apresentada na área pequena existe um ganho no desempenho nas provas com dicas válidas $(551 \mathrm{~ms})$ e um custo nas provas com dicas válidas para área $(592 \mathrm{~ms}) \mathrm{em}$ relação às provas sem dica $(572 \mathrm{~ms})$. Quando os estímulos são apresentados na área grande, tanto as provas com dicas validas $(580 \mathrm{~ms})$ como as provas com dicas válidas para área (652ms) apresentam ganhos significantes em relação às provas sem dica (670ms).

Comparando os resultados obtidos nesta condição experimental com aqueles obtidos em tratamentos equivalentes do experimento anterior, podemos observar neste experimento um aumento na diferença entre os TRs obtidos com estímulos apresentados nas posições indicadas e estímulos apresentados em posições intermediárias. No Experimento 1, a diferença entre os TRs obtidos nas provas com dicas válidas e válidas para área foi de $7 \mathrm{~ms}$, no Experimento 2 essa diferença foi de $72 \mathrm{~ms}$. Nas provas com estímulos apresentados na área menor, essas diferenças foram de 26 e $41 \mathrm{~ms}$, respectivamente. Ou seja, a apresentação gradual acentuou a diferença entre o tempo de processamento de estímulos apresentados em locais indicados e nas posições intermediárias. Este resultado pode ser explicado tanto por um modelo de focos atencionais múltiplos, como por um modelo de foco único guiado pela ativação sensorial presente nas diferentes posições espaciais, mas é incompatível com um modelo de foco único de tamanho flexível, tal como o do zum da lente (Eriksen \& Yeh, 1985; Eriksen \& St. James, 1986).

Nas provas com dica global múltipla apresentada na área pequena existe apenas um custo nas provas com dicas inválidas $(597 \mathrm{~ms})$ em relação às provas sem dica $(575 \mathrm{~ms})$, mas as provas com dicas válidas para área (587ms) não apresentam ganho. $\mathrm{O}$ mesmo acontece quando os estímulos são apresentados na área maior; nas provas com dicas inválidas (691ms), o custo é significante em relação às provas sem dica $(652 \mathrm{~ms})$, mas não existe ganho nas provas com dicas válidas $(652 \mathrm{~ms})$. Nas provas com dica global o desempenho não varia em função da validade da dica quando os estímulos são apresentados na área menor, mas existe um ganho significante nas provas com dicas válidas (634ms) em relação às provas sem dica $(659 \mathrm{~ms})$ quando os estímulos são apresentados na área maior.

A análise da taxa de respostas incorretas (em torno de 5\% do total) replica as características básicas da análise do TR e não será apresentada em detalhes. $\mathrm{O}$ coeficiente de correlação entre a taxa de respostas incorretas e o TR é igual a 0,60, assim podemos descartar a existência de um conflito entre rapidez e precisão em nossos resultados.

De acordo com um modelo de foco único, que cobriria toda a área indicada, e não apenas posições específicas, a informação proporcionada pela dica local deveria ter proporcionado 
ganho tanto nas provas com dicas válidas como nas provas com dicas válidas para área. Isto acontece quando os estímulos são apresentados na área maior, mas não quando são apresentados na área menor. Neste caso existe um ganho associado à correta localização dos estímulos nas dicas válidas, mas existe custo nas provas válidas apenas para área, quando os estímulos são apresentados entre as posições indicadas. Ou seja, o modelo de foco atentivo único parece ter certa validade para áreas grandes, mas não para áreas pequenas. A ausência de ganho na situação em que os estímulos são apresentados em uma área pequena entre as posições indicadas (dicas válidas para área), assemelha-se aos resultados obtidos por Kramer e Hahn (1995). Os estímulos apresentados entre as posições indicadas, têm seu tempo de processamento aumentado, sugerindo que os locais indicados receberam mais recursos atencionais, tal como se poderia supor com base num modelo de foco atencional múltiplo. Uma vez que neste experimento os estímulos foram apresentados de maneira gradual, a ativação sensorial, desencadeada pelo aparecimento abrupto dos estímulos, não parece oferecer a explicação mais adequada para os resultados.

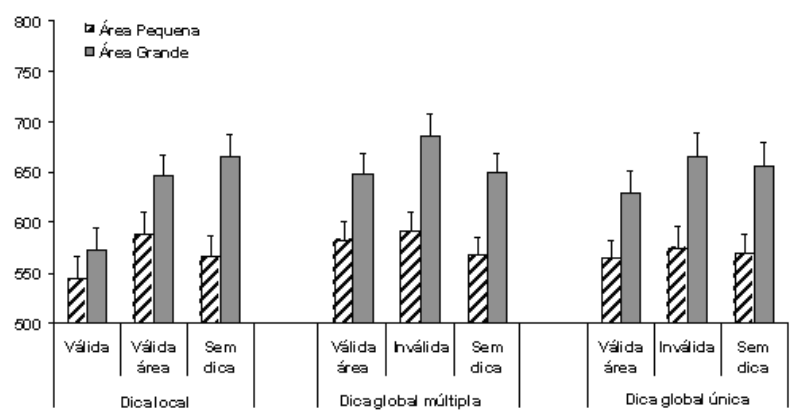

Figura 4. TRs médios (e erro padrão da média) nas condições com dica Local, Global múltipla e Global única em função da área na qual foram apresentados estímulos e da validade da dica.

Nas provas com as dicas globais inválidas, múltipla e única, existe um custo associado à mudança no tamanho da área coberta pelas dicas e pelos estímulos, mas não existe ganho nas provas em que a área é informada corretamente pela dica válida. Estes resultados sugerem que o tamanho do foco atentivo deve ser reajustado ao tamanho da área na qual são apresentados os estímulos nas provas com dicas inválidas, o que estaria de acordo com um modelo de foco único de tamanho flexível (Castiello \& Umiltà, 1990; Eriksen \& St. James, 1986; Eriksen \& Yeh, 1985). No entanto, a ausência de ganho nas provas com dicas válidas mostra que esta explicação não é completamente adequada aos resultados deste experimento, principalmente quando se observa que existe ganho nas condições equivalentes com dica válida para área, tanto no Experimento 1 como nas provas com dica local deste segundo experimento. Estas diferenças entre o efeito das dicas obtidas nos dois experimentos sugerem que o emprego da atenção depende não só das características físicas dos estímulos apresentados numa prova específica, mas também das características do contexto experimental em que estes estímulos são apresentados.

\section{Discussão Geral}

Neste estudo avaliamos as características do foco da atenção visual utilizando o paradigma de dicas múltiplas em uma tarefa de busca visual. Nossos resultados sugerem que as características do foco atentivo variam em função do tipo de dica e do tamanho da área coberta pelos estímulos. Quando os estímulos são apresentados na área maior, a informação proporcionada pela dica local contribui para um ganho no desempenho, mesmo quando os estímulos são apresentados em posições intermediárias, mas neste caso o ganho é menor do que aquele obtido com provas válidas. Este resultado não é compatível com um modelo de foco único, que prevê ganhos de mesma magnitude tanto quando os estímulos são apresentados nas posições indicadas como quando são apresentados nas posições intermediárias. Quando os estímulos são apresentados na área menor, existe um cuzdica válida para área nos experimentos 1 e 2). Este resultado também permite argumentar contra o modelo de foco único e sugere que um modelo baseado em um processo de ativação sensorial ou baseado em focos múltiplos seria mais adequado.

Vários estudos têm mostrado que o foco atentivo se "ajusta" ao tamanho da área definida pela dica (Castiello \& Umiltà, 1990; Eriksen \& St. James, 1986; Eriksen \& Yeh, 1985; Turatto\& cols., 2000), também existem evidências de que mudanças na escala espacial da atenção, introduzidas por mudanças no tamanho da área indicada, modulam a atividade neural em áreas primárias do córtex visual (Luo, Greenwood \& Parasuraman, 2001). Neste estudo também encontramos um custo nas provas em que a dica informa incorretamente a área na qual os estímulos serão apresentados (dica inválida). No Experimento 1, esse custo é significante quando os estímulos são apresentados na área maior, sugerindo que o custo estaria associado à abertura do foco, mas não à sua contração (estímulos apresentados na área menor). Este resultado poderia ser esperado com base num modelo de zum. Mas, no Experimento 2, existe um custo tanto para a abertura como para o fechamento do foco, contrariando esse modelo.

Embora as dicas locais possam ter um efeito global, por exemplo, nas provas em que os estímulos são apresentados entre as posições indicadas, esse efeito é diferente daquele obtido nas provas com dicas globais. Nestas provas não existe ganho quando a dica é válida, mas existe custo quando é inválida. Este custo parece estar associado à mudança no tamanho do foco atentivo. $\mathrm{O}$ fato de ocorrer um custo tanto nas provas em que as dicas informam uma área menor do que aquela na qual serão apresentados os estímulos, como quando indicam uma área maior sugere um possível mecanismo de focalização da atenção, equivalente ao efeito do tamanho da dica (Eriksen \& St. James, 1986; Eriksen \& Yeh, 1985). Mas, o fato de não termos obtido um efeito facilitador para estas dicas globais sugere que o ganho no desempenho está associado à informação sobre o local em que serão apresentados os estímulos e não em informações mais gerais sobre a área.

A forma de apresentação dos estímulos, abrupta no Expe- 
rimento 1 e gradual no Experimento 2, não afetou qualitativamente os resultados, mas aumentou a magnitude dos ganhos associados às dicas válidas no segundo experimento. Enquanto que no Experimento 1, a diferença entre o TR das provas válidas e válidas para área foi de $21 \mathrm{~ms}$ para a área pequena e de $7 \mathrm{~ms}$ para a área grande, o ganho no experimento 2 foi de 41 e $71 \mathrm{~ms}$, respectivamente. Ou seja, a apresentação gradual das dicas e estímulos, que teoricamente diminuiu a ativação sensorial atribuível ao aparecimento abrupto, facilitou a detecção do alvo nos pontos indicados. $\mathrm{O}$ fato do ganho obtido com as provas válidas no segundo experimento não ser afetado pelos IDEs utilizados sugere que a contribuição da ativação sensorial pode ter sido substituída pela orientação voluntária do foco atencional para as posições indicadas.

De maneira geral, o modelo de focos múltiplos e o modelo de ativação sensorial são os que melhor explicam nossos resultados. Mas, mesmo esses modelos precisariam ser revistos para dar conta da interação entre validade da dica e tamanho da área indicada. Pois, se podem explicar o custo associado na detecção dos estímulos apresentados entre os locais indicados nas áreas pequenas dos experimentos 1 e 2, não dão conta do ganho encontrado quando os estímulos são apresentados na área maior. Recentemente, Müller e Kleinschmidt (2004) apresentaram evidências fisiológicas de inibição ao redor de localizações atendidas ao mostrar que a atividade neural em áreas visuais periféricas, vizinhas às áreas atendidas, mostrava uma supressão de atividade. E, que áreas mais distantes voltavam a apresentar um aumento de ativação. Mais recentemente, Müller, Mollenhauer, Rösler e Kleinschmidt (2005) apresentaram evidências comportamentais de que a ativação atencional segue o mesmo perfil da distribuição do chapéu mexicano. Se considerarmos que a atenção em cada um dos focos múltiplos obedece à distribuição do chapéu mexicano, poderemos explicar o custo associado às provas locais válidas na área pequena e o ganho nas áreas grandes, como função da distância entre dois focos atencionais. Se nas áreas pequenas, um estímulo apresentado entre duas posições indicadas está localizado nas áreas de inibição de um ou de ambos os focos atentivos, poderemos supor que esse estímulo seria inibido. Nas provas realizadas nas áreas grandes, um estímulo apresentado entre as posições indicadas estaria localizado a distâncias maiores dos pontos indicados, possivelmente em uma área de ativação periférica. Esse estímulo não seria inibido, mas receberia uma ativação menor do que os estímulos apresentados nos locais indicados, levando ao pequeno ganho observado nas provas válidas para área com os estímulos apresentados na área maior. Estas suposições estão sendo investigadas.

\section{Referências}

Bahcall, D. O. \& Kowler, E. (1999). Attentional interference at small spatial separations. Vision Research, 39, 71-86.

Bichot, N. P, Cave, K.R. \& Pashler, H. (1999). Visual selection mediated by location: Feature-based selection of noncontiguous locations. Perception \& Psychophysics,_61, 403-423.
Castiello, U. \& Umiltà, C. (1990). Size of attentional focus and efficiency of processing. Acta Psychologica, 73, 195-209.

Cave, K. R. \& Bichot, N. P. (1999). Visuospatial attention: Beyond a spotlight model. Psychonomic Bulletin and Review, 6, 204-223.

Eriksen, C. W. \& St. James, J. D. (1986). Visual attention within and around the field of focal attention: A zoom lens model. Perception \& Psychophysics, 40, 225-240.

Eriksen, C. W. \& Yeh, Y. Y. (1985). Allocation of attention in the visual field. Journal of Experimental Psychology: Human Perception \& Performance, 5, 583-595.

Galera, C., Lopes, E. J. \& von Grünau, M. (2001). Stimulus segmentation in the visual search task. Perception \& Psychophysics, 62, 505-516.

Heinze, H. J., Luck, S. J, Münte, T. F., Gös, A., Mangun, G. R. \& Hillyard, S. A. (1994). Attention to adjacent and separate positions in space: An electrophysiological analysis. Perception \& Psychophysics, 56, 42-52.

Kramer, A. F. \& Hahn, S. (1995). Splitting the beam: Distribution of attention over noncontiguous regions of the visual field. Psychological Science, 6, 381-386.

LaBerge, D. \& Brown, V. (1989). Theory of attentional operation in shape identification. Psychological Review, 96, 101-124.

Luo, Y. J.,Greenwood, P. M. \& Parasuraman, R. (2001). Dynamics of the spatial scale of the visual attention revealed by brain eventrelated potential. Cognitive Brain Research, 12, 371-381.

Müller, N. G. \& Kleinschmidt, A. (2004). The attentional 'spotlight's' penumbra: center-surround modulation in striate cortex. Neuroreport, 15, 977-980.

Müller, N. G., Mollenhauer M., Rösler, A. \& Kleinschmidt, A. (2005). The attentional field has a Mexican hat distribution. Vision Research, 45, 1129-1137.

Posner, M. I. (1980). Orienting of attention. Quarterly Journal of Experimental Psychology, 32, 3-25.

Posner, M.I., Snyder, C.R.R. \& Davidson, B.J. (1980). Atention and the detection of signals. Journal of Experimental Psychology: General, 109, 160-174.

Richard, C.M., Wright, R.D. \& Ward, L.M. (2003). Goal-driven modulation of stimulus-driven attentional capture in multiple-cue displays. Perception \& Psychophysics, 65, 939-955.

Scharlau, I. (2004). Evidence for split foci of attention in a priming paradigm. Perception \& Psychophysics, 66, 988-1002.

Schneider, W., Eschman, A. \& Zuccolotto, A. (2002). E-Prime User's Guide. Pittsburgh: Psychology Software Tools Inc.

Turatto, M., Benso, F., Facoetti, A., Galfano, G., Mascetti, G.G. \& Umiltà, C. (2000). Automatic and voluntary focusing of attention. Perception \& Psychophysics, 62, 935-952.

Wright, R.D. (1994). Shifts of Visual Attention to Multiple Simultaneous Location Cues. Journal of Experimental Psychology: Human Perception and Performance, 48, 205-217.

Wright, R.D., \& Richard, C.M. (2003). Sensory mediation of stimulusdriven attentional capture in multiple-cue displays. Perception \& Psychophysics, 65, 925-938.

Yantis, S. \& Jonides, J. (1984). Abrupt visual onsets and selective attention: evidence from visual search. Journal of Experimental Psychology Human Perception and Performance, 10, 601-621.

Recebido em 15.06.2005

Primeira decisão editorial em 12.06.2006 Aceito em 13.11.2006 